\title{
STATISTICAL MODEL FOR PSEUDO-MOVING OBJECTS RECOGNITION IN VIDEO SURVEILLANCE SYSTEMS
}

\author{
B. Vishnyakov, A. Egorov, S. Sidyakin, I. Malin, and Y. Vizilter \\ State Research Institute of Aviation Systems (FGUP GosNIIAS), Moscow, Russia - \\ (vishnyakov, aegorov, sersid, imalin, viz)@gosniias.ru
}

\section{Commission III}

KEY WORDS: Outlier Objects, Pseudomoving Objects, Statistical Criteria, Bayesian Integration, Logistic Regression

\begin{abstract}
:
This paper considers a statistical approach to define pseudo-moving (false) objects in video surveillance systems by constructing systems of hypothesis with the criteria based on statistical behavioral particularities. The obtained results are integrated in two ways: using the Bayes' theorem or the logistic regression. FAR-FRR curves are plotted for each system of hypothesis and also for the decision rule. The results of the proposed methods are obtained on test video databases.
\end{abstract}

\section{INTRODUCTION}

Nowadays efforts of modern video surveillance systems developers are aimed at the intellectualization of as many as possible functions to facilitate the operator's work. Such functions include so-called «rules» that generate video analytic events when certain situations occur, as, for example, the intersection of a line by an object, the movement in the forbidden zone, the movement in prohibited direction, etc. It is important to minimize the number of false alarms, because if this number is comparable with the number of the faithful positives, then the operator's attention is getting weaker quickly. In this state operator begins to skip all the events, often not thoroughly enough. Finally, it leads to skipping of important or even dangerous events.

Along with the actual moving objects, the so-called "pseudomoving” objects appear very often on video sequences. These objects usually include camera noise, glare and reflections, shadows, swaying branches and foliage. Such objects, appearing in the rules areas, generate a large number of false events. Therefore, to reduce the number of false alarms, it is necessary to understand, which of moving objects are valid in a scene, and which are false (pseudo-moving).

We assume that the problem of motion detection is already solved (Elgammal, 2000; Stauffer, 1999; Vishnyakov, 2012) and we have a list of moving objects or areas with confirmed motion. For example, we can use algorithms of motion detection and object tracking, proposed in papers (Elhayek, 2012; Hosmer, 2002; McHugh, 2008). The most common methods for false objects detection, described in literature, use objects or background brightness and geometrical features (Stauffer, 2000; Torr, 1993). Also, there is a number of methods for shade and shadow detection (Sanin, 2012). The difference of this work is application of the statistical methods for the pseudo-moving objects detection without taking the brightness and color object features and a background model into account. In our approach we consider only the information about the movement nature. Also it should be noted, that our model for pseudo-moving objects recognition can be used jointly with any other methods of false object detection.

In this paper, we consider a set of statistical features specific to pseudo-moving objects. Hypotheses systems are constructed for the proposed features. In each system, we suggest the null hypothesis - the object is pseudo-moving («false»), and the alternative - the object is a valid moving object. After hypothesis verification the final conclusion about the object type in each system is made. The results are integrated on Bayes ' theorem, where the posterior probabilities of all hypotheses are counted on video training set. In addition, we propose the second way of the criteria integration using logistic regression. The quality evaluation of proposed methods on a large amount of real video data from public databases PETS and ETISEO is presented.

\section{STATISTICAL APPROACH FOR PSEUDO-MOVING OBJECTS DETERMINATION}

In the general case, the moving objects have the following most commonly used attributes to identify them: shape, size, texture, brightness distribution, contrast, movement speed. However, for various reasons, it is not always possible to classify an object according to these criteria: because of insufficient amount of information, because of the technical imperfection of equipment or due to the nature of the observed scene. In this paper we propose the following distinctive features, which can be used for the pseudo-moving and valid objects classification:

1. The presence or absence of a trend component in the object motion model;

2. The ratio of the object detection time to its presence time; 
3. The number of direction changes.

For each property the following hypothesis system is constructed:

1. The null hypothesis $H_{k}^{0}=$ \{the object is pseudo-moving $\}$;

2. The alternative hypothesis $H_{k}^{1}=\{$ the object is valid $\}$.

Here the parameter $k=1,2,3$ is the number of the hypotheses system.

The hypotheses system: the trend component in the model of the object motion

Usually in motion analysis, the number of incoming frame is used as the time moment. Therefore, per unit of time $t$ it is possible to take either the frame number, or a frame timestamp. Let $x(t), y(t)$ be the coordinates of the object's center of mass. Let us consider a linear model of the object motion on a finite time interval:

$$
\begin{aligned}
& x(t)=a_{1} t+b_{1}+\varepsilon_{1}(t) \\
& y(t)=a_{2} t+b_{2}+\varepsilon_{2}(t) \\
& t \in\left[t_{1}, t_{2}\right]
\end{aligned}
$$

where

$$
\begin{aligned}
& a_{i}, b_{i}-\text { unknown model parameters } \\
& \varepsilon_{i}-\text { observation errors, } \\
& i=1,2 \\
& t_{1}, t_{2} \text { - moments of time during the object's life }
\end{aligned}
$$

We choose the linear model as an object movement model, because of its simplicity and sufficiency for trend component estimation. Movement trend is considered to be $a_{i}, i=1,2$ parameter in mathematical model (1).

Based on the video sequences analysis from public databases we found that most of the pseudo moving objects do not have a pronounced trend component, in contrast to the actual objects. We suggest calculating statistics $Z_{1}$ using estimated parameters $\widehat{a_{l}}, i=$ 1,2 from mathematical model (1) normalized by estimated meansquare deviations of observation errors $\widehat{\sigma}_{l}, i=1,2$ for the assessment of the object movement trend:

$$
Z_{1}=\max \left(\frac{\hat{a}_{1}}{\hat{\sigma}_{1}}, \frac{\hat{a}_{2}}{\hat{\sigma}_{2}}\right)
$$

This criterion allows classifying effectively valid and pseudomoving objects of almost any type (perhaps, except reflections) of the movement nature. Fig. 1 shows a histogram of the sample statistics $Z_{1}$ for real-moving objects and pseudo-moving objects.

\subsection{The hypotheses system: missing object observations}

Very often different algorithms for tracking of moving objects have a heightened sensitivity to contrast. When there is insufficient contrast between the object and background, the socalled losses of the objects observations are possible. The object is considered as missing, if at some moment of time (on some frame) it is not possible to detect it. Observation losses are typical for such pseudo moving objects as swaying branches or camera noise. As the statistics $Z_{2}$ we suggest using the following expression:

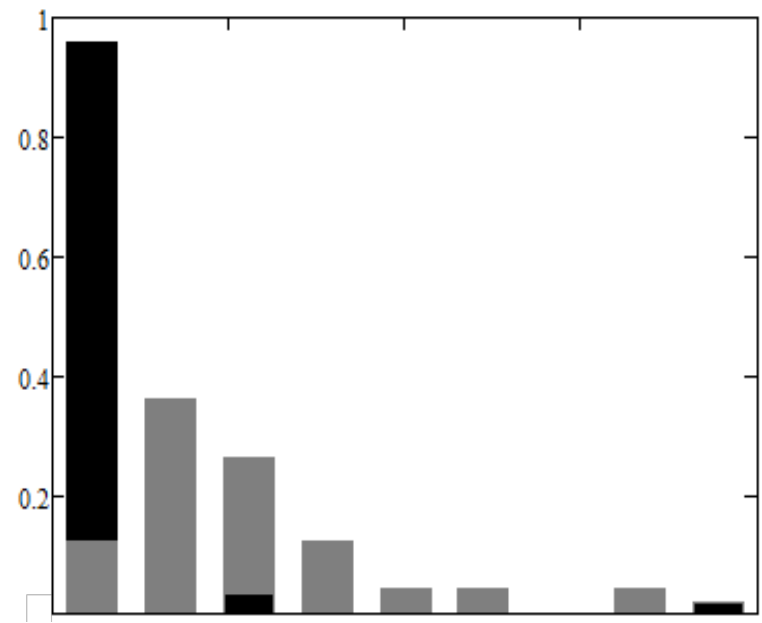

Figure 1. The histogram of the statistics $\boldsymbol{Z}_{\mathbf{1}}$ for real-moving objects - gray columns and pseudo-moving objects - black columns.

$$
Z_{2}=\frac{N_{\text {tracked }}}{N_{\text {overall }}}
$$

where $N_{\text {tracked }}=$ the number of frames on which the object was detected

$N_{\text {overall }}=$ the total frames number of the object's life from the moment of its first detection to the moment of making decision on the object type.

Fig. 2 shows the histogram of the statistics sample implementation $Z_{2}$ for real-moving objects and pseudo-moving objects.

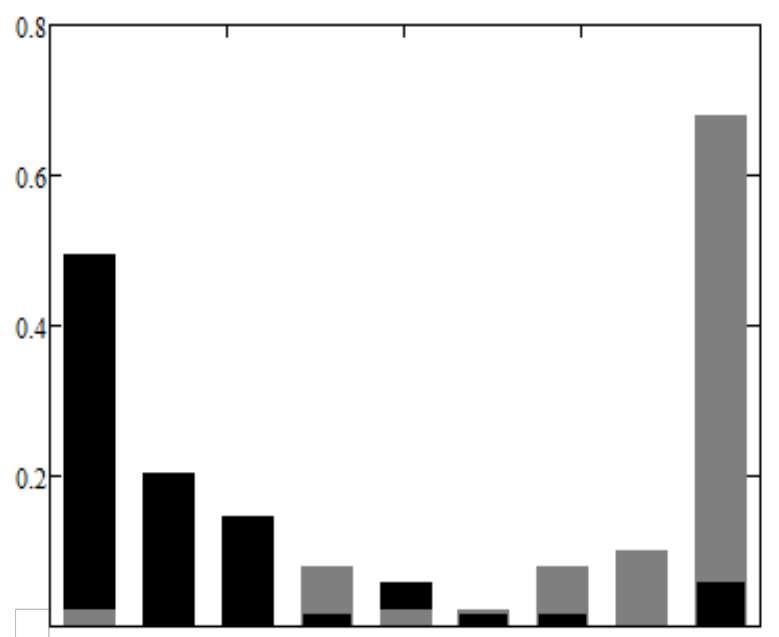

Figure 2. The histogram of the statistics $Z_{2}$ for real-moving objects - gray columns and pseudo-moving objects - black columns. 
It is clear from the histogram that with any values of the separating threshold the probability of type II error will be different from zero. This is explained by the fact that some pseudo-moving objects can be detected almost $N_{\text {overall }}$ frames. These objects include, for example, shadows or reflections. The value of $Z_{2}$ statistic for such objects, as can be seen from fig. 2, can be the same as for the real-moving objects. In such a case, the criterion will not be able to classify them correctly.

\subsection{The hypotheses system: changes of object movement direction.}

Based on the test sequences analysis we found out the possibility to identify pseudo-moving objects using the number of changes in the movement. Such objects as, for example, the swaying branches of trees often change the direction of movement during their lifetime. Usually it does not refer to real moving objects. The movement trajectory changes are used as the statistics $Z_{3}$.

$$
Z_{3}=N_{\text {turns }}
$$

where $N_{\text {turns }}$ - the number of trajectory turns.

We assume that the object has changed the movement direction, if $\left(V_{i} \cdot V_{i-1}\right)<0$ (turn angle is more than $90^{\circ}$ ), where $V_{i}-$ is the object movement vector over time $\left(t_{i}, t_{i-1}\right), \quad V_{i-1}$ - the object movement vector during the time $\left(t_{i-1}, t_{i-2}\right)$, the operation - the scalar product.

Fig. 3 shows the histogram of the statistics sample implementation $Z_{3}$ for real-moving objects and pseudo-moving objects.

As it can be seen from the histogram, some pseudo-moving objects cannot be correctly classified according to the criterion due to the nature of their movement, if they do not change the direction. Such objects may include, for example, glares and some reflections. At the same time, some real moving objects can move with the frequent changes in the trajectory (this objects behavior is called as «loitering»), which also does not allow classifying them correctly.

It should be noted that we considered other variants of verifying the «swaying» fact, including defining of the significance of a periodic component in the model. However, proposed simple criterion, based on a calculation of object velocity, showed the best ability for classification of false objects along with significantly higher performance, compared to other similar criteria.

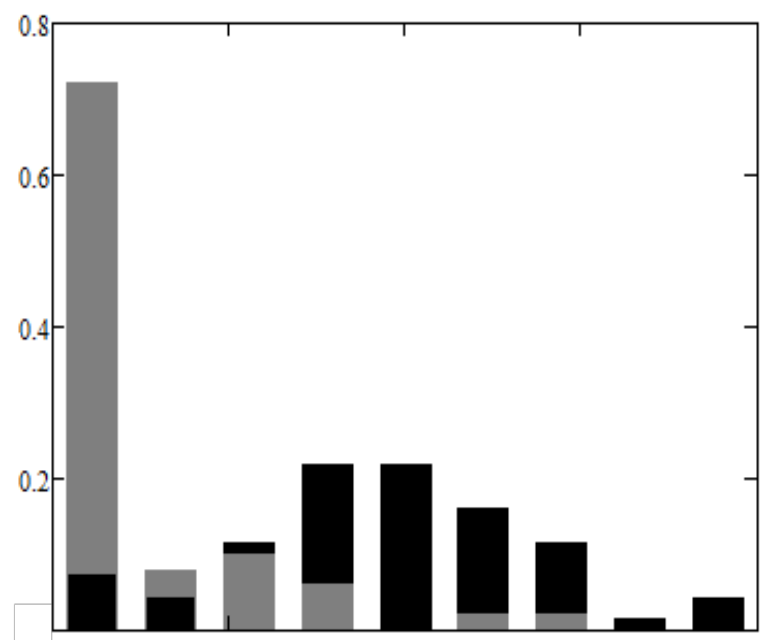

Figure 3. The histogram of the statistics $Z_{3}$ for real-moving objects - gray columns and pseudo-moving objects - black columns.

\section{THE INTEGRATION CRITERION ON THE BASIS OF BAYES' THEOREM}

Totally, three statistical criteria were constructed, based on three sets of hypotheses:

1. Hypothesis $H_{1}^{0}$ : the object is pseudo moving, if it has no pronounced trend component. Its alternative $-H_{1}^{1}$.

2. Hypothesis $H_{2}^{0}$ : the object is pseudo moving, if it is unsustainably detected. Its alternative $-H_{2}^{1}$.

3. Hypothesis $H_{3}^{0}$ : the object is pseudo moving, if it often changes its direction. Its alternative $-H_{3}^{1}$.

\begin{tabular}{|c|c|c|c|c|}
\hline & & \multicolumn{3}{|c|}{$\begin{array}{c}\text { Accepted } \\
\text { hypothesis }\end{array}$} \\
\hline Probability & Value & $H_{1}^{0}$ & $H_{2}^{0}$ & $H_{3}^{0}$ \\
\hline$\hat{P}\left(E_{0} \mid H_{1}^{0} H_{2}^{0} H_{3}^{0}\right)$ & 0.9991 & + & + & + \\
\hline$\hat{P}\left(E_{0} \mid H_{1}^{0} H_{2}^{0} H_{3}^{1}\right)$ & 0.987 & + & + & - \\
\hline$\hat{P}\left(E_{0} \mid H_{1}^{0} H_{2}^{1} H_{3}^{0}\right)$ & 0.983 & + & - & + \\
\hline$\hat{P}\left(E_{0} \mid H_{1}^{1} H_{2}^{0} H_{3}^{0}\right)$ & 0.962 & - & + & + \\
& & & & \\
\hline$\hat{P}\left(E_{0} \mid H_{1}^{0} H_{2}^{1} H_{3}^{1}\right)$ & 0.273 & + & - & - \\
\hline$\hat{P}\left(E_{0} \mid H_{1}^{1} H_{2}^{0} H_{3}^{1}\right)$ & 0.137 & - & + & - \\
\hline
\end{tabular}




\begin{tabular}{|c|c|c|c|c|}
\hline$\hat{P}\left(E_{0} \mid H_{1}^{1} H_{2}^{1} H_{3}^{0}\right)$ & 0.108 & - & - & + \\
\hline$\hat{P}\left(E_{0} \mid H_{1}^{1} H_{2}^{1} H_{3}^{1}\right)$ & 0.0008 & - & - & - \\
\hline
\end{tabular}

Table 1. Assessment of the system effectiveness.

To construct the final classifier we introduce the following events: $E_{0}$ - the object is pseudo moving; $E_{1}$ - the object is not pseudo moving. The probabilities of these events are evaluated on the training sample and take the following values:

$$
\hat{P}\left(E_{0}\right), \hat{P}\left(E_{1}\right)=1-\hat{P}\left(E_{0}\right),
$$

Probabilities $\quad \hat{P}\left(H_{1}^{i} \mid E_{m}\right), \hat{P}\left(H_{2}^{j} \mid E_{m}\right), \hat{P}\left(H_{3}^{k} \mid E_{m}\right) \quad$ where $i, j, k, m=0,1$ were also estimated on the training sample.

Then, using Bayes' theorem and (5), as well as the fact that the statistical criteria are independent by nature (hypothesis about the criteria correlations were verified and accepted), the formula for the calculation of the classification probabilities estimates takes the following form:

$$
\begin{aligned}
& \hat{P}\left(E_{0} \mid H_{1}^{i} H_{2}^{j} H_{3}^{k}\right)= \\
& \frac{\hat{P}\left(H_{1}^{i} \mid E_{0}\right) \hat{P}\left(H_{2}^{j} \mid E_{0}\right) \hat{P}\left(H_{3}^{k} \mid E_{0}\right) \hat{P}\left(E_{0}\right)}{\sum_{m=0,1} \hat{P}\left(H_{1}^{i} \mid E_{m}\right) \hat{P}\left(H_{2}^{j} \mid E_{m}\right) \hat{P}\left(H_{3}^{k} \mid E_{m}\right) \hat{P}\left(E_{m}\right)}
\end{aligned}
$$

where $i, j, k, m=0,1$

Calculated values for all possible sets of $i, j, k$ are shown in table 1. Based on estimates of the system effectiveness, the decisive rule is that the object is considered as pseudo-moving in case if at least two null hypotheses were accepted.

\section{THE CRITERIA AGGREGATION USING LOGISTIC REGRESSION}

Logistic regression is one of the linear classification methods [8]. We chose this method of classification, because it is very robust to outliers. In this model, the objects are represented as the $n$ dimensional feature vectors. In our case, the feature vector will consist of statistics described in section 2: $Z_{1}, Z_{2}, Z_{3}$. We consider $n=4$ (an extra bias is added, the value of which is equal to 1 for all objects, which provides a hyperplane of a general view, not only passing through the coordinates origin). Values 1 and 0 serve as a class mark, depending on whether the object belongs to the pseudo-moving objects class or not. Thus, a training set $T=$ $\left\langle x_{i}, y_{i}\right\rangle$, where $i \in 1 . . m$ - the object number in the test sample, $x_{i} \in R^{n}$ - feature vector, and $y_{i} \in\{0,1\}$ - a class mark, corresponding to this object. A penalty function in logistic regression is given:

$$
\begin{aligned}
& J(T, \theta)=\frac{1}{m} \sum_{i=1}^{m}\left(-y_{i} \cdot \log \left(h_{\theta}\left(x_{i}\right)\right)-\left(1-y_{i}\right) \cdot \log \left(1-h_{\theta}\left(x_{i}\right)\right)\right) \\
& h_{\theta}(x)=\frac{1}{1+\exp \left(-\theta^{T} \cdot x\right)}
\end{aligned}
$$

Machine learning task is to find the optimal vector of parameters $\theta^{*} \in R^{n}$, minimizing the cost function:

$$
\theta=\arg \min J(T, \theta)
$$

Thus, this classifier is looking for a hyperplane in the space $R^{n}$, separating the two classes objects, which is optimal in the logistic regression statement. Fig. 4 shows the separating hyperplane for valid and false objects found by proposed algorithms on one of the videos from public database PETS.

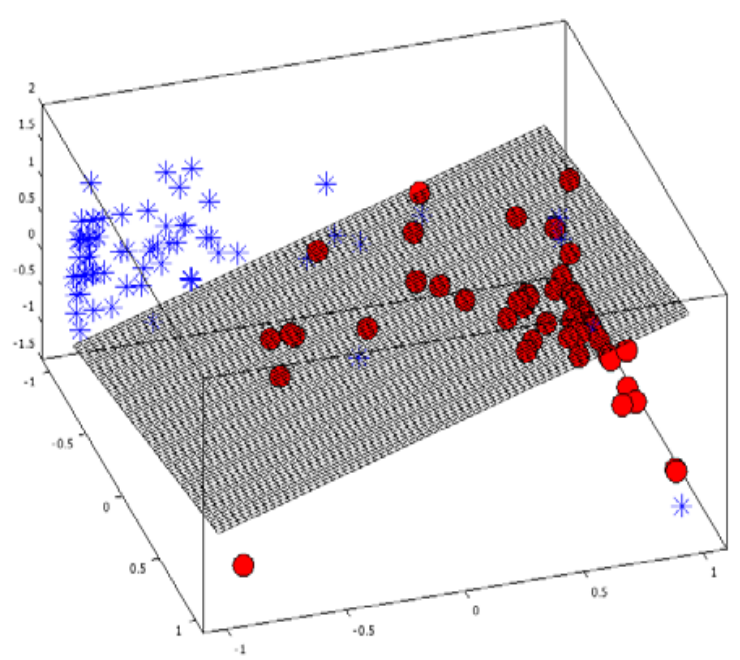

Figure 4. Separating hyperplane for true (circles) and false (stars) objects for ETISEO video.

The decisive rule is verification of the condition $\theta^{T} \cdot x \geq 0$, which means that the objects will be considered as belonging to the class "pseudo moving" (with the mark of a class 1 ). If the condition is not met, then the object will belong to the class of real (valid) objects (labeled with 0 ).

\section{CLASSIFICATON ALGORITHMS TESTING}

Algorithms of fast automatic pseudo-moving objects classification, based on developed methods, were tested on publicly available databases - PETS, ETISEO and CANDELA. The cross-validation methods were used for assessment of confusion matrix and the errors probabilities of the first and the second kinds. The original objects sample was divided into $\mathrm{q}=5$ noncrossing subsets of approximately equal cardinality, each of which in turn became a reference, other four - learning. Confusion matrix elements were calculated and summed for q control subsets. The procedure of splitting the original sample to q subsets and confusion matrix calculation was carried out $\mathrm{t}=5$ times, whereupon the average of the values for these partitioning options was computed. Also, we configured the sensitivity of the basic moving object detector so that many outliers were detected. 
Table 2 shows confusion matrix for the method, based on Bayesian criteria integration.

\begin{tabular}{|c|c|c|c|}
\hline & \multicolumn{3}{|c|}{ Estimated } \\
\hline ర్d & Type & Real object & False \\
\hline هั & Real object & $476(0.899)$ & $54(0.101)$ \\
\hline 苛 & False & $51(0.073)$ & $641(0.927)$ \\
\hline
\end{tabular}

Table 2. Confusion matrix for Bayesian integration.

To determine the quality of decision rule in the classification manner, fig. 5 shows the false acceptance and false rejection rates (hereinafter FAR-FRR) curves for the criteria individually and for the decision rule for integration using logistic regression.

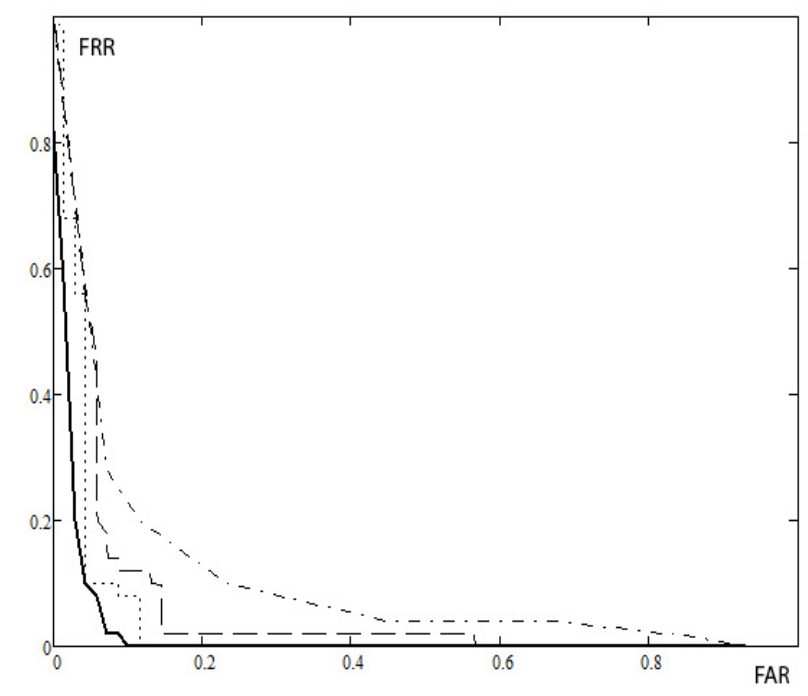

Figure 5: FAR-FRR curves for the logistic regression (upper dotted curves for statistical criteria individually, lower solid - for the decision rule).

Table 3 shows the confusion matrix for the method, based on logistic regression.

\begin{tabular}{|c|c|c|c|}
\hline & & \multicolumn{2}{|c|}{ Estimated } \\
\hline \multirow{3}{*}{ 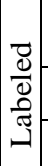 } & Type & Real object & False \\
\hline & Real object & $491(0.927)$ & $39(0.073)$ \\
\hline & False & $37(0.053)$ & 655 (0.947) \\
\hline
\end{tabular}

Table 3. Confusion matrix for logistic regression.

Fig. 6 shows an example of the proposed algorithms use to cut-off pseudo-moving objects.
As it can be seen from the testing results, linear classifier based on logistic regression model showed slightly better results than the classifier based on Bayesian integration.

In addition, it should be noted that we do not compare our results to other ones, because for now we have not found any similar papers on the subject.

\section{CONCLUSIONS}

In this paper, we present an approach for the pseudo-moving objects recognition in video analysis tasks. Testing on a large volume of real video data showed high efficiency of the considered methods (type I and type II errors around 0.07). The offered technique for the determination of pseudo-moving objects on video sequences can be used in many systems of the situational video analysis of dynamically changing scenes, such as the systems of video surveillance of public places, the systems of transport flows estimation, security systems and other engineering systems of similar purpose.

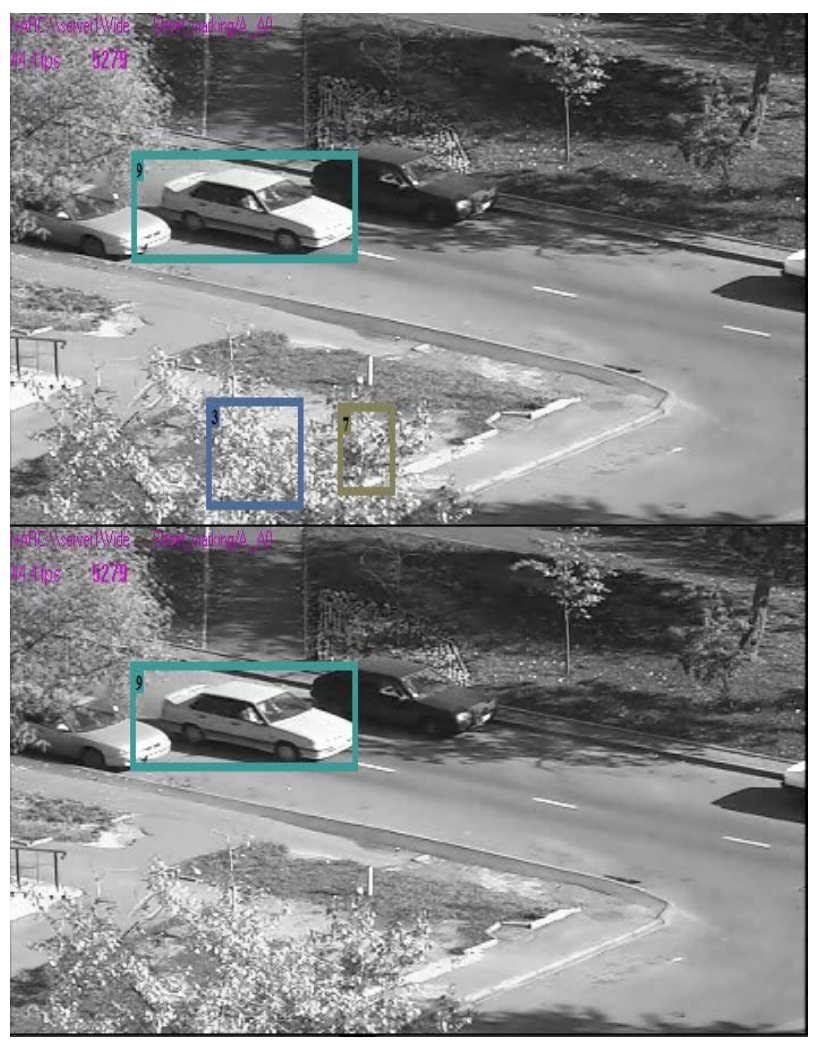

Figure 6. On the picture above you can see two pseudo-moving objects, on the picture below these objects are determined as false and removed.

\section{REFERENCES}

Elgammal A., Harwood D. and Davis, L., 2000. Non-parametric model for background subtraction. In Proc. Eur. Conf. on Computer Vision, Lect. Notes Comput. Science. 1843, pp. 751767. 
Elhayek A., Stoll C. and others, 2012. Spatio-temporal Motion Tracking with Unsynchronized Cameras. Proceedings of the 2012 IEEE Conference on Computer Vision and Pattern Recognition, pp. 1870-1877.

Hosmer David W. Lemeshow Stanley, 2002. Applied Logistic Regression, 2nd ed. New York, Chichester, Wiley.

McHugh M., Konrad J. and others, 2008. Motion Detection With False Discovery Rate Control. IEEE Inter. Conf. on Image Processing, pp. 873-876.

Sanin A., Sanderson C., Lovell B. C., 2012. Shadow Detection: A Survey and Comparative Evaluation of Recent Methods. Pattern Recognition, Vol. 45, No. 4, pp. 1684-1695.

Stauffer C. and Grimson W. E. L., 1999. Adaptive background mixture models for real-time tracking. In Proc. Int. Conf. on Computer Vision and Pattern Recognition, Vol. 2, IEEE, Piscataway, NJ.

Stauffer C, Grimson W. E. L, 2000. Learning Patterns of Activity Using Real-Time Tracking. IEEE Transactions on Pattern Analysis and Machine Intelligence, 22(8), pp. 747-757.

Torr P. H. S., Murray D. W., 1993. Outlier Detection and Motion Segmentation. Proceedings of SPIE, 2059, pp. 432-443.

Vishnyakov B., Vizilter Y., Knyaz V., 2012. Spectrum-Based Object Detection And Tracking Technique For Digital Video Surveillance. International Archives of the Photogrammetry, Remote Sensing and Spatial Information Sciences, pp. 579-583.

Video database "Pefomance Evaluation of Tracking and Surveillance (PETS)” http://www.cvg.rdg.ac.uk/slides/pets.html.

Video database "Video Understanding Evaluation project ETISEO” http://www-sop.inria.fr/orion/ETISEO/. 